\title{
Risk perceptions, nutrition, and physical activity among South Asian women in the US: Does history of gestational diabetes mellitus (GDM) matter?
}

\author{
Amita Vyas*, Nitasha Chaudhary, Kalpana Ramiah, Grace Kathryn Douglasss \\ Department of Prevention and Community Health, School of Public Health and Health Services, The George Washington University, \\ Washington DC, USA; \\ *Corresponding Author: avyas@gwu.edu
}

Received 13 September 2012; revised 16 October 2012; accepted 27 October 2012

\begin{abstract}
Objective: This study examines risk perceptions, nutrition practices, and physical activity among ever pregnant South Asian American women, and explores differences by history of GDM, a significant risk factor for the development of type 2 diabetes. Methods: The Diabetes Prevention Study (DPS) recruited a convenience sample of South Asian adults living in the metropolitan Washington DC region. Specific eligibility criteria included English proficiency; having at least one child between the ages of 5 and 15; no current diagnosis of type 2 diabetes mellitus (T2DM); and having a family history of T2DM. The present study utilizes a subset of the DPS dataset and includes 109 ever pregnant women, including $58 \%$ with a history of GDM. Results: Mean scores for perceptions of risk showed that both worry and personal control are slightly greater than "neutral" with 3.5 out 5 . Therefore, women worry about T2DM yet also perceive personal control for their risk. $40.2 \%$ of all respondents use ghee (clarified butter) to cook meals and $41.7 \%$ re-use cooking oil. $35 \%$ of respondents report no physical activity in an average week. Only $39.8 \%$ of women meet the recommended guidelines for adults in the US There are no significant differences between women with or without a history of GDM for nutrition practices and physical activity. Conclusions: This study adds to the literature on GDM and missed opportunities for the prevention of future T2DM. Future research ought to explore knowledge levels on T2DM during and after pregnancy, as well as what types of intervenetions would be effective and acceptable to South Asian women.
\end{abstract}

Keywords: Gestational Diabetes; South Asian; Health Behavior; Prevention; Type 2 Diabetes

\section{INTRODUCTION}

Gestational diabetes mellitus (GDM) is defined as a "glucose intolerance with the onset or first recognition of pregnancy” [1,2]. According to the American Diabetes Association (ADA), it affects nearly three to eight percent of all pregnant women [3], with approximately 135,000 cases diagnosed in the US alone [4]. Although previous estimates have found that Asian-Indians, who comprise the largest subset of South Asians, have some of the highest rates of GDM in the US with $11 \%$ compared to $4 \%$ among whites [5], little research has been conducted with this ethnic group. The Diabetes Prevention Study (DPS), a community survey of South Asian Americans living in the Washington DC region, was conducted to better understand risk perceptions and health behaviors related to type 2 diabetes mellitus (T2DM). The present manuscript is a sub-study of DPS focusing on ever pregnant women to assess differences in risk perceptions, nutrition practices, and physical activity between women with and without a history of GDM.

There are approximately 2.7 million South Asians living in the US comprising of individuals with family origins from Afghanistan, Bangladesh, Bhutan, India, the Maldives, Nepal, Pakistan, and Sri Lanka [6]. Although the South Asian population in the US has significantly increased in the last several decades, there has been limited data and research on health-related perceptions and behaviors among this growing population. More specifically, this is a community more likely to develop T2DM; to be diagnosed at younger ages; and to experience more T2DM related complications than the general population [7-9]. There are several hypotheses with ample evidence explaining the significant prevalence of T2DM among South Asian Americans, mostly centering on genetic 
susceptibility, increased incidence of abdominal obesity, and insulin resistance [9]. Furthermore, the Diabetes Prevention Program (DPP) estimated the cumulative incidence of T2DM was 38.4\% for women with a history of GDM compared with only $25.7 \%$ for women without a history of GDM [10]. Although there is an increased risk for developing T2DM among women with a history of GDM, almost no studies have focused solely on South Asian women in the US

In the last decade, population-based studies have found ethnicity to be a risk factor for GDM with Hispanic and Asian women at an increased risk [5,10,11]. A study conducted by Hedderson et al., concluded that there is an $80 \%$ increased risk of GDM among Asian Indian women and this increased risk is also prevalent in several other Asian subgroups (Chinese, Southeast Asian and Filipina) and in Mexicans and Pacific Islanders [5]. Additional studies examining Asian subgroups provide further evidence that migrant populations have a higher prevalence of GDM as compared to the prevalence in their native countries [5,12-15], suggesting that GDM is influenced by both environmental and behavioral factors, as well as genetic predisposition.

A cohort study conducted in Canada by the Ontario Ministry of Health followed over one million women aged 20 - 49 years with in-hospital live births. Study results indicated that the age- adjusted prevalence of GDM was $41 \%$ higher among Chinese and 145\% higher among South Asians compared to their white counterparts [16]. Of these women, there was also an increased incidence of T2DM in the first 4 to 6 months postpartum pointing to the importance screening all women with a history of GDM. The ADA recommends screenings at 6 to 12 weeks postpartum by either fasting plasma glucose or with a oral glucose test $[17,18]$. In addition, women should be re-evaluated every year if results come out abnormal, and every three years if there is no impaired glucose tolerance or impaired fasting glucose detected [17]. While the ADA and the American Congress of Obstetrics and Gynecologists (ACOG) have set guidelines on screening postpartum, particularly with women who have GDM, findings suggest that many women are not screened for T2DM after delivery [19]. Although it is evident that GDM is an increased risk factor for T2DM, many women with GDM are unaware of this risk [20]. Jones et al. found that women with previous GDM have a $20 \%$ to $50 \%$ chance of developing type 2 diabetes in the next 5 to 10 years, and the prevalence may be higher depending on race/ethnicity [21].

Studies reveal that many women with a history of GDM do not only lack the knowledge of diabetes, but more so do not fully understand the relationship between how lifestyle behaviors and developing T2DM [19,22]. Further, theoretical health models suggest that risk per- ception is a critical determinant of health behavior [23]. Of the $90 \%$ of women in an academic managed plan who had GDM, only $16 \%$ believed they were at an increased risk for T2DM [24]. Moreover, in ethnic-specific women, specifically Pima Indians [25] and Mexican Indians [26], women did not believe that GDM increased their risk for developing T2DM postpartum. A 2009 review of the literature found that women with a history of GDM often had lower perceptions of risk for T2DM, as well as poorer levels of physical activity and nutrition [21]. To help eliminate and/or reduce the risk, interventions must address other significant contributing factors including perceived advantages and barriers to healthy lifestyle behaviors including healthy diet and exercise [21,24].

In addition to increased postpartum screening, recommendations have been put forth by the ADA and ACOG on lifestyle modifications for women with a history of GDM [3,4]. Modifications in diet, exercise, and weight reduction and/or maintenance have specifically been shown to decrease the progression of T2DM in several populations including Asians [27,28]. Moreover, although Asian-American women have the highest risk for GDM compared with other racial/ethnic groups, GDM risks and rates vary within Asian subgroups quite significantly, and therefore more attention is needed to fully understand specific populations such as South Asian women [29]. The present study utilizes data from the Diabetes Prevention Study (DPS), a community survey of South Asian adults with a family history of T2DM. The underlying purpose of DPS was to better understand risk perceptions and health behaviors in order to develop a culturally salient lifestyle intervention for this high-risk ethnic population in the US This paper examines risk perceptions, nutrition practices, and physical activity among ever pregnant South Asian American women, and explores differences by history of GDM, a risk factor for future T2DM diagnosis.

\section{RESEARCH DESIGN AND METHODS}

\subsection{Sample and Procedures}

The Diabetes Prevention Study (DPS) recruited a convenience sample of South Asian adults living in the Washington DC metropolitan region. Specific eligibility criteria included English proficiency; having at least one child between the ages of 5 and 15; no current diagnosis of T2DM; and having a family history of T2DM. For purposes of this study, South Asian is defined as individuals from the following countries: Bangladesh, Bhutan, India, the Maldives, Nepal, Pakistan, and Sri Lanka. The present study utilizes a subset of the DPS dataset and includes 109 ever pregnant women, including $58 \%$ with a history of GDM.

The research team collaborated with eleven South 
Asian faith-based and cultural organizations in the Washington DC region for recruitment and data collection. Between July 2011 and March 2012, participants were recruited into the study. Consent was obtained prior to survey administration. To ensure privacy and reduce reporting bias, surveys were administered via individual laptop computers. Study participants completed the survey in English and were given a \$25 gift card for their efforts in completing the survey. Upon survey completion, the data were stored in an encrypted file only to be read by the survey design software, SNAP surveys (SNAP Surveys Ltd., 2012).

Recruitment at sites yielded a $92 \%$ response rate, and the majority of those who declined to participate stated "not enough time" as the primary reason. People of Indian descent represent $54.7 \%$ of the South Asian population in the Washington DC region and therefore the majority of organizations (approximately 90\%) in the region are tailored to this community. Significant efforts were made to reach out to all non-Indian focused organizations in the region for data collection, however $87 \%$ of participants in the study are of Indian origin.

\subsection{Instrument and Measures}

The survey instrument for the present study gathered information on demographic characteristics, risk perceptions, nutrition practices and physical activity, and took approximately 15 - 20 minutes to complete. Survey questions were adapted from the 2009 National Health and Nutrition Examination Survey (NHANES) [30] and Risk Perception Survey for Developing Diabetes (RPS-DD) [22].

Demographic characteristics include variables on age, marital status, parity, and country of origin. In addition, immigration status was captured by US born, US citizen, and number of years of living in the US For women with a history of GDM, additional questions were asked including: 1) number of pregnancies diagnosed with GDM, 2) type of treatment (insulin or medication versus none), and 3) whether the GDM pregnancy resulted in a c-section.

Perceptions of risk include two scales measuring "worry" and "personal control”. Both scales are based on reliable and validated measures from E.A. Walker’s 2009 Risk Perception survey [22]. Participants were asked if they strongly agree, agree, neither agree nor disagree, disagree or strongly disagree with a series of statements. Several statements were reverse coded to ensure that $1=$ low worry/control and $5=$ high worry/control. Three items measure "worry" as follows: 1) I worry about my family members getting diabetes, 2) I am very concerned about getting diabetes, and 3) Worrying about diabetes is very upsetting to me. The Cronbach's alpha for the worry scale in this sample is 0.71. Six items measure “personal control” as follows: 1) Type 2 diabetes can be prevented, 2) I have little or no control over risks to my health, 3) If I am going to get diabetes, there is not much I can do about it, 4) My personal efforts help control my risk of getting diabetes, 5) Getting diabetes is all a chance of fate that is meant to be and unavoidable, and 6) Genetics and family history have everything to do with getting diabetes. The Cronbach's alpha for the personal control scale in this sample is 0.58 .

Several questions from the 2009 NHANES [30] were adapted to measure nutrition related behaviors. Participants were asked how often in an average week they: 1) skip breakfast, 2) eat potato chips, nacho chips, hot mix, crackers or popcorn, 3) eat fast food, 4) add butter, margarine, ghee (clarified butter originated in South Asia) or oil to bread, potatoes, rice or vegetables at the table, 5) eat sweets more than once a day, and 6) drink 16 oz. or more of non-diet soda, fruit juice/punch a day. All statements share the following response options: most of the time, some of the time, and rarely/never. Two additional nutrition-related questions were developed based on cultural cooking practices among South Asians as follows: 1) Do you or other family members in your home use ghee (clarified butter) in order to cook your meals (yes/no)? and 2) Do you or other family members in your home save and/or re-use the oil previously used to cook meals (yes/no)?

A series of questions on physical activity to capture type and amount of time were asked. Participants were asked separately about vigorous and moderate activity for at least 30 minutes continuously, as well as number of days per week. Examples of vigorous activity include running, bicycling, swimming, exercise classes, and basketball. Examples of moderate activity include brisk walking, yoga, and badminton. Participants could report engaging in both vigorous and moderate activity, and the amount of days differed for each. The responses were categorized as follows: No physical activity = no vigorous or moderate activity; Low physical activity = vigorous activity less than 5 days per week only or moderate activity less than 5 days per week only; Medium physical activity = vigorous activity less than 5 days and moderate activity 5 or more days per week or vigorous and moderate activity less than 5 days per week; High physical activity = vigorous and moderate activity 5 or more days per week or vigorous activity 5 or more days per week only. Given that both medium and high levels of physical activity meet the recommended guidelines for physical activity in the US, these two categories were collapsed to provide more meaningful interpretation of the data.

\subsection{Analysis}

Given the sample size for this study ( $\mathrm{n}=109$ ), much 
of the analysis is descriptive and focuses on an overall description of the study population, including overall mean scores for risk perceptions and overall percentages for nutrition and physical activity variables. Some of the variable categories were collapsed to increase the usefulness of the information. Bivariate analysis was conducted to examine any significant differences between women with a history of GDM and those without.

\section{RESULTS}

The study sample consists of 109 ever pregnant females with 63 (57.8\%) having a history of GDM. As shown in Table 1, the mean age for the sample is 40.2

Table 1. Characteristics of study participants by GDM history, diabetes prevention study $(\mathrm{n}=109)$.

\begin{tabular}{|c|c|c|c|}
\hline & No GDM History $(\mathrm{N}=46)$ & GDM History $(\mathrm{N}=63)$ & Total $(\mathrm{N}=109)$ \\
\hline & $\%(\mathrm{~N})$ & $\%(\mathrm{~N})$ & $\%(\mathrm{~N})$ \\
\hline Mean Age (years) & $40.3 \pm 6.2$ & $40.1 \pm 8.5$ & $40.2 \pm 7.6$ \\
\hline \multicolumn{4}{|l|}{ Marital status } \\
\hline Single & $2.2(1)$ & $0.00(0)$ & $0.9(1)$ \\
\hline Married & $95.6(44)$ & $96.8(61)$ & $96.3(105)$ \\
\hline Divorced & $2.2(1)$ & $3.2(2)$ & $2.8(3)$ \\
\hline \multicolumn{4}{|l|}{ Parity } \\
\hline One & $36.4(16)$ & 36.5 (23) & 36.5 (39) \\
\hline Two & $52.3(23)$ & $50.8(32)$ & $51.4(55)$ \\
\hline Three+ & $11.3(5)$ & $12.7(8)$ & $12.1(13)$ \\
\hline \multicolumn{4}{|l|}{ Born in the US } \\
\hline Yes & $8.7(4)$ & $15.9(10)$ & $12.8(14)$ \\
\hline No & $91.3(42)$ & $84.1(53)$ & $87.2(95)$ \\
\hline \multicolumn{4}{|l|}{ US citizen Yes No } \\
\hline & $69.6(32)$ & $66.7(42)$ & $67.9(74)$ \\
\hline & $30.4(14)$ & $33.3(21)$ & $32.1(35)$ \\
\hline \multicolumn{4}{|l|}{ Years in US } \\
\hline less than 5 years & $9.5(4)$ & $7.5(4)$ & $8.4(8)$ \\
\hline $6-10$ years & $16.7(7)$ & $26.4(14)$ & $22.1(21)$ \\
\hline 11 - 20 years & $45.2(19)$ & $35.9(19)$ & $40.0(38)$ \\
\hline 21 years or more & $28.6(12)$ & $30.2(16)$ & 29.5 (28) \\
\hline \multicolumn{4}{|l|}{ Country of Origin } \\
\hline India & 84.8 (39) & $88.3(53)$ & $86.8(92)$ \\
\hline Other & $15.2(7)$ & $11.7(7)$ & $13.2(14)$ \\
\hline \multicolumn{4}{|l|}{ No. of pregnancies diagnosed with Gestational Diabetes } \\
\hline One & $\mathrm{n} / \mathrm{a}$ & $71.4(45)$ & \\
\hline Two & $\mathrm{n} / \mathrm{a}$ & $27.0(17)$ & \\
\hline Three & $\mathrm{n} / \mathrm{a}$ & $1.6(1)$ & \\
\hline Type of pharmaceutical treatment for GDM Insulin or oral medication & $\mathrm{n} / \mathrm{a}$ & $49.7(24)$ & \\
\hline No pharmaceutical treatment & $\mathrm{n} / \mathrm{a}$ & $59.3(36)$ & \\
\hline \multicolumn{4}{|l|}{ C-section } \\
\hline Yes & $\mathrm{n} / \mathrm{a}$ & $42.6(27)$ & \\
\hline No & $\mathrm{n} / \mathrm{a}$ & $55.7(34)$ & \\
\hline
\end{tabular}

No significant differences by GDM history. 
years and the majority of respondents are currently married (96.3\%), have two children (51.4\%), and their country of origin is India (86.8\%). Only $12.8 \%$ of participants were born in the US but $67.9 \%$ are US citizens with $89.5 \%$ having lived in the US for 11 or more years. Of the $57.8 \%$ who have ever been diagnosed with GDM, $71.4 \%$ were diagnosed with one pregnancy, $49.7 \%$ were treated with either insulin or medication; and $42.6 \%$ delivered via cesarean. There are no significant differences by GDM history and demographic characteristics.

Table 2 presents mean scores for perceptions of risk. As shown, the overall mean scores for both "worry" and personal control are slightly greater than "neutral" with 3.5 ( 1 = low worry/control; 5 = high worry/control). Therefore, women worry about T2DM yet also perceive personal control for their risk. For the "worry" scale, women with a history of GDM, have a higher mean score of 3.6 versus 3.3, though non-significant. For "personal control”, women with a history of GDM have a slightly higher mean score of 3.6 versus 3.5, but also non-significant.

Table 2 also presents nutrition practices by GDM status. As shown, $40.2 \%$ of all respondents use ghee (clarified butter) to cook meals; and $41.7 \%$ re-use cooking oil. Overall, 40.2\% of report skipping breakfast most/some of the time; $52.8 \%$ eat "fried" snacks most/some of the time; $53.3 \%$ add butter/margarine/ghee to food at the table; and $59.4 \%$ eats sweets more than once a day. There are no significant differences between women with or without a history of GDM for any of

Table 2. Mean scores for perceptions of risk, percentages for nutrition, and physical activity by GDM history, diabetes prevention survey $(n=109)$.

\begin{tabular}{|c|c|c|c|}
\hline & \multicolumn{3}{|c|}{ History of GDM } \\
\hline & No $(\mathrm{N}=46)$ & Yes $(\mathrm{N}=63)$ & Total $(\mathrm{N}=109)$ \\
\hline \multicolumn{4}{|l|}{ Perceptions of risk } \\
\hline Worry Scale (mean score) & 3.3 & 3.6 & 3.5 \\
\hline Control scale (mean score) & 3.5 & 3.6 & 3.5 \\
\hline \multicolumn{4}{|l|}{ Nutrition behavior } \\
\hline \multicolumn{4}{|l|}{ Uses ghee } \\
\hline Yes & $43.5(20)$ & $37.7(23)$ & $40.2(43)$ \\
\hline No & $24.2(20)$ & $35.5(38)$ & $59.8(64)$ \\
\hline \multicolumn{4}{|l|}{ Re-uses cooking oil } \\
\hline Yes & $43.5(20)$ & $40.3(25)$ & $41.7(45)$ \\
\hline No & $56.5(26)$ & $34.3(37)$ & $58.3(63)$ \\
\hline $\begin{array}{l}\text { Eats regular potato chips, nacho chips, hot mix, crackers, } \\
\text { or popcorn most/some of the times }\end{array}$ & $54.6(24)$ & $51.6(32)$ & $52.8(56)$ \\
\hline Rarely/never & $45.4(20)$ & $48.4(30)$ & $47.23(50)$ \\
\hline Eats fast food most/some of the times & $34.1(15)$ & $43.6(27)$ & $39.6(42)$ \\
\hline Rarely/never & $65.9(29)$ & $56.4(35)$ & $60.4(64)$ \\
\hline $\begin{array}{l}\text { Adds butter, margarine, ghee or oil to bread, potatoes, } \\
\text { rice or vegetables at the table most/some of the time }\end{array}$ & $62.2(28)$ & $46.7(28)$ & $53.3(56)$ \\
\hline Rarely/never & $37.8(17)$ & $53.3(32)$ & $46.7(49)$ \\
\hline Eats sweets more than once a day most/some of the time & $62.2(28)$ & $57.4(35)$ & $59.4(63)$ \\
\hline Rarely/never & $37.8(17)$ & $42.6(26)$ & $40.6(43)$ \\
\hline $\begin{array}{l}\text { Drinks } 16 \text { oz. or more of non-diet soda, fruit juice/punch } \\
\text { or Kool-Aid a day Most/some of the times }\end{array}$ & $13.3(6)$ & $26.7(16)$ & $21.0(22)$ \\
\hline Rarely/never & $86.7(39)$ & $73.3(44)$ & $79.0(83)$ \\
\hline \multicolumn{4}{|l|}{ Physical Activity } \\
\hline None & $32.6(14)$ & $36.7(22)$ & $35(36)$ \\
\hline Low & $16.3(7)$ & $51.2(22)$ & $25.2(26)$ \\
\hline High & $51.2(22)$ & $31.7(19)$ & $39.8(41)$ \\
\hline
\end{tabular}


these nutrition-related behaviors.

With respect to physical activity, 35\% of respondents report no physical activity in an average week. Only $39.8 \%$ of women report "high" physical activity which represents the recommended guidelines for adults in the US.

Women with a history of GDM diabetes are less likely to engage in "high" physical activity (31.1\% versus $51.2 \%)$, though this was not significant.

\section{DISCUSSION}

This study explored South Asian American women's risk perceptions for T2DM, nutrition practices, and physical activity level. Further, given the increased risk for developing T2DM among women with a history of GDM, the study examined potential differences between women with and without a history of GDM. South Asians have one of the highest rates of T2DM and studies have consistently found that South Asian women are at high-risk for GDM. And yet, very little is known about lifestyle factors in this ethnic population of women. This study is the first to specifically focus on South Asian women in the Washington DC region, home to one of the largest populations of South Asians in the US.

Overall, participants reported that they worry about developing T2DM and simultaneously reported personal control over developing T2DM. It is possible that although women worry, they also have some level of knowledge regarding how it can be "controlled" or prevented. Surprisingly, there were not significant differences between women with a history of GDM and those without. Given the elevated risk of developing T2DM for women with a history of GDM, future studies should explore risk perceptions immediately postpartum and then again at a future time. It is possible that women with a history of GDM may perceive greater risk immediately after a pregnancy and that perception may decrease over time, particularly if follow-up interventions and screening is not in place.

Overall, women in this study engage in cultural nutriation practices that can increase one's risk for developing T2DM. Approximately $40 \%$ of women reported use of ghee and/or re-using cooking oil, both of which can increase trans-fat content when preparing meals. Furthermore, participants reported other "unhealthy" nutritional practices such as skipping breakfast, eating chips, adding butter/margarine, and eating sweets more than once a day. Interestingly, across all of these nutrition practices, there were not any significant differences between women with and without a history of GDM. Physical activity levels among women in this study were quite low, with $60.2 \%$ of women not meeting the recommended guidelines for weekly physical activity.

Despite the increased risk for developing T2DM among women with a history of GDM, this study found that they were not engaging in healthier behaviors compared to women without a history of GDM. This study has revealed an important need and gap in services and interventions.

First, future work is needed to better understand why South Asian American women in general are not engageing in better nutritional practices and physical activity. Both gender and culture play a central role in decisions surrounding food choice, preparation of meals, and acceptable types of physical activity. These domains must be explored in an effort to develop T2DM prevention programs aimed at South Asian women in the US, and must incorporate and address culture and gender.

Second, this study adds to the literature on GDM and missed opportunities for the prevention of future T2DM. Postpartum screening must be extended to include more coordinated care and follow-up between obstetricians, pediatricians and primary care providers, all of whom interact with women during the postpartum period. A report from the TRIAD Study found that although postpartum screening for T2DM has increased among women with a history of GDM, significant gaps still remain and many women are still being missed [31].

Future research ought to explore knowledge levels on T2DM during and after pregnancy, as well as what types of interventions would be effective and acceptable to South Asian women. The Diabetes Prevention Program (DPP) found that the incidence of T2DM was reduced by $50 \%$ among women with a history of GDM who were randomized into an intensive lifestyle intervention or metformin therapy [10]. The significance of DPP and its potential to be adapted and tailored for South Asian women in the United States is incredibly important and more attention must be given to how cultural norms can be integrated into prevention programs such as these. Bandyopadhyay et al. have provided examples of how South Asian culture is central to pregnancy [32]. The authors point out that after a GDM diagnosis, South Asian women in the US women are often caught between cultural beliefs and controlling their GDM. Most often, South Asian women adhere to a more sedentary lifestyle and increased caloric intake during pregnancy which may be at odds with provider recommendations.

GDM and T2DM are public health challenges facing women in the United States and both future research as well as interventions aimed at the prevention of T2DM must advance the knowledgebase on specific racial and ethnic groups. As diverse communities continue to grow in the United States, research and programs must continue to explore cultural frameworks and their mechanisms in providing both risk and protection for T2DM.

It is important to note that there are several methodological limitations to this study. First, the study was lim- 
ited by its cross-sectional design and it was not powered adequately for multivariate analysis, and therefore the findings are primarily descriptive in nature. Second, given the larger numbers of community organizations targeting Asian-Indians, the study sample consists mostly of Asian Indian and does not represent other South Asian subgroups. In addition, the questionnaire was developed in English only and, therefore, non-English speaking South Asians were not included in the study. Finally, the time since last pregnancy was not assessed as well as whether participants received postpartum information or education regarding future risks for T2DM, both of which may influence current nutrition practices and physical activity. Despite these limitations, this study recruited participants from a high risk ethnic group that has been given little attention, and provides important insights that will guide the next series of studies and interventions to address the prevention of T2DM among South Asian American women with a history of GDM.

\section{CONCLUSION}

The public health community has made significant efforts in understanding the risks associated with developing T2DM among women with a history of GDM, and yet studies consistently show missed opportunities for interventions post-pregnancy. As evident in the data illustrated in this study, South Asian women need to understand the risks of gestational diabetes and how to prevent future T2DM through dietary modification and physical activity. In more recent years, considerable interest and work has gone into identifying and raising awareness related to health disparities for specific minority populations in the US However, for those groups and others, much more needs to be done. As the South Asian community has grown over the last several decades, so too have the challenges and pressures faced by many. Yet stereotypes, such as "model minority" and "wealthy and healthy," continue to perpetuate. However, these paradoxes are not observed across all areas of health. The health needs of South Asian women, especially those with a history of GDM, must become part of the women's health agenda and integrated into current and future public health initiatives aimed at the prevention of type 2 diabetes.

\section{ACKNOWLEDGEMENTS}

This publication was supported by Award Number UL1RR031988/ UL1TR000075 from the NIH National Center for Advancing Translational Sciences. Its contents are solely the responsibility of the authors and do not necessarily represent the official views of the National Center for Advancing Translational Sciences or the National Institutes of Health. The authors acknowledge the support and cooperation of local community and faith-based organizations, and we are grateful to all the community members who participated in this project.

\section{REFERENCES}

[1] Jovanovic, L. and Pettitt, D.J. (2001) Gestational diabetes mellitus. The Journal of the American Medical Association, 286, 2516-2518. doi:10.1001/jama.286.20.2516

[2] Mukerji, G., Chiu, M. and Shah, B.R. (2012) Impact of gestational diabetes on the risk of diabetes following pregnancy among Chinese and South Asian women. Diabetologia, 55, 2148-2153.

[3] American Diabetes Association (2006) Diagnoses and classification of diabetes mellitus. Diabetes Care, 29, 543548.

[4] American College of Obstetricians and Gynecologists (2001) ACOG practice bulletin: Assessment of risk factors for preterm birth: Clinical management guidelines for obstetrician-gynecologists. Obstetrics and Gynecology, 98, 709-716.

[5] Hedderson, M.M., Darbinian, J.A. and Ferrara, A. (2010) Disparities in the risk of gestational diabetes by race-ethnicity and country of birth. Paediatric and Perinatal Epidemiology, 24, 441-448.

doi:10.1111/j.1365-3016.2010.01140.x

[6] The World Bank (2010) South Asian: Countries. http://web.worldbank.org/WBSITE/EXTERNAL/COUN TRIES/SOUTHASIAEXT/0,,menuPK:158850 pagePK:1 46748 piPK:146812 theSitePK:223547,00.html

[7] Mohan, V., Vijayachandrika, V., Gokulakrishnan, K., Anjana, R.M., Ganesan, A., Weber, M.B. and Narayan, K.M. (2010) A1C cut points to define various glucose intolerance groups in Asian Indians. Diabetes Care, 33, 515-519. doi:10.2337/dc09-1694

[8] Tirodkar, M.A., Baker, D.W., Makoul, G.T., Khurana, N., Paracha, M.W. and Kandula, N.R. (2011) Explanatory models of health and disease among South Asian immigrants. Journal of Immigrant and Minority Health, 13, 385-394.

[9] Greenlaugh, P.M. (1997) Diabetes in British South Asians: Nature, nurture, and culture. Diabetic Medicine, 14, 10-18. doi:10.1002/(SICI)1096-9136(199701)14:1<10::AID-DI A282>3.0.CO;2-B

[10] Ratner, R.E., Christophi, C.A., Metzger, B.E., Dabelea, D., Bennett, P.H., Pi-Sunyer, X., Fowler, S. and Kahn, S.E. (2008) Prevention of diabetes in women with a history of gestational diabetes: Effects of metformin and lifestyle interventions. The Journal of Clinical Endocrinology \& Metabolism, 93, 4774-4779. doi:10.1210/jc.2008-0772

[11] Hunsberger, M., Rosenberg, K.D. and Donatelle, R.J. (2012) Racial/ethnic disparities in gestational diabetes mellitus: Findings from a population based survey. Women's Health Issues, 20, 323-328. doi:10.1016/j.whi.2010.06.003

[12] Misra, A. and Ganda, O.P. (2007) Migration and its impact on adiposity and type 2 diabetes. Nutrition, 23, 696708. doi:10.1016/j.nut.2007.06.008

[13] Zargar, A.H., Sheikh, M.I., Bashir, M.I., Masoodi, S.R., 
Laway, B.A., Wani, A.I., et al. (2006) Prevalence of gestational diabetes mellitus in Kashmiri women from the Indian subcontinent. Diabetes Research and Clinical Practice, 66, 139-145. doi:10.1016/j.diabres.2004.02.023

[14] Franco, L.J. (1996) Diabetes in Japanese-Brazilians influence of the acculturation process. Diabetes Research and Clinical Practice, 34, S51-S57.

[15] Fujimoto, W.Y., Bergstrom, R.W., Boyko, E.J., Leonetti, D.L., Newell, M.L.L. and Wahl, P.W. (1995) Susceptibility to development of central adiposity among populations. Obesity Research, 3, 179S-186S. doi:10.1002/j.1550-8528.1995.tb00461.x

[16] Shah, B.R., Chiu, M., Amin, S., Ramani, M., Sadry, S. and Tu, J.V. (2010) Surname lists to identify South Asian and Chinese ethnicity from secondary data to Ontario, Canada: A validation study. BMC Medical Research Methodology, 10, 42. doi:10.1186/1471-2288-10-42

[17] Bentley-Lweis, R., Levkoff, S., Steube, A. and Sweely, E.W. (2008) Gestational diabetes mellitus: Postpartum opportunities for the diagnosis and prevention of type 2 diabetes mellitus. Nature Clinical Practice Endocrinology \& Metabolism, 4, 552-558. doi:10.1038/ncpendmet0965

[18] American Diabetes Association (2004) Gestational diabetes mellitus. Diabetes Care, 27, S88-S90. doi:10.2337/diacare.27.2007.S88

[19] Kim, C., Tabaei, B.F., Burk, R., McEwen, L.N., Lash, R.W., Johnson, S.L., Schwartz, K.L., Bernstein, S.T. and Herman, W.H. (2006) Missed opportunities for type 2 diabetes mellitus screening among women with a history of gestational diabetes mellitus. American Journal of Public Health, 96, 1643-1648. doi:10.2105/AJPH.2005.065722

[20] Fisher, E.B., et al. (2002) Behavioral science research in the prevention of diabetes: Status and opportunities. Diabetes Care, 25, 599-606. doi:10.2337/diacare.25.3.599

[21] Jones, E.J., Roche, C.C. and Appel, S.J. (2009) A review of the health beliefs and lifestyle behaviors of women with previous gestational diabetes. The Association of Women's Health, Obstetric and Neonatal Nurses, 38, 516-526.

[22] Walker, E.A., Mertz, C.K., Kalten, M. and Flynn, J. (2003) Risk perception for developing diabetes: Comparative risk judgments of physicians. Diabetes Care, 26, 25432548. doi:10.2337/diacare.26.9.2543

[23] SpJane, N.K. and Becker, M.H. (1984) The health belief model: A decade later. Health Education Quarterly, 11, 147. doi:10.1177/109019818401100101

[24] Kim, C., et al. (2007) Risk perception for diabetes among women with histories of gestational diabetes mellitus. Diabetes Care, 30, 2281-2286. doi:10.2337/dc07-0618

[25] Smith-Morris, C.M. (2005) Diagnostic controversy: Gestational diabetes and the meaning of risk for Pima Indian women. Medical Anthropology, 24, 145-177. doi:10.1080/01459740590933902

[26] Kieffer, E.C., et al. (2002) Perspectives of pregnant and postpartum Latino women ondiabetes, physical activity, and health. Health Education Behavior, 29, 542-556.

[27] Pan, X.R., et al. (1997) Effects of diet and exercise in preventing NIDDM in people with impaired glucose tolerance. The Da Qing IGT and diabetes study. Diabetes Care, 20, 537-544. doi:10.2337/diacare.20.4.537

[28] Tucker, M.E. (2003) Prescribe diet, exercise, after gestational diabetes (many will develop type 2 diabetes). $\mathrm{Ob}$ stetrics \& Gynecology News, 38, 10.

[29] Chu, S.Y., Abe, K., Hall, L.R., Kim, S.Y., Njoroge, T. and Qin, C. (2009) Gestational diabetes mellitus: All Asians are not alike. Preventive Medicine, 20, 265-268. doi:10.1016/j.ypmed.2009.07.001

[30] Centers for Disease Control and Prevention (2009) National health and nutrition examination survey. http://www.cdc.gov/nchs/data/nhanes/nhanes_09_10/FCB S_f.pdf

[31] Ferrara, A., Peng, T. and Kim, C. (2009) Trends in postpartum diabetes screening and subsequent diabetes and impaired fasting glucose among women with histories of gestational diabetes mellitus. Diabetes Care, 32, 269-274. doi:10.2337/dc08-1184

[32] Bandyopadhyay, M., Small, R., Davey, M., Oats, J.J.N., Forster, D.A. and Alyward, A. (2011) Lived experience of gestational diabetes mellitus among immigrant South Asian women in Australia. Australian and New Zealand Journal of Obstetrics and Gynecology, 51, 360-364. doi:10.1111/j.1479-828X.2011.01322.x 